\title{
Ideal strength and phonon instability of strained monolayer materials
}

\author{
Eric B. Isaacs * and Chris A. Marianett \\ Department of Applied Physics and Applied Mathematics, Columbia University, New York, NY 10027
}

(Dated: June 21, 2022)

\begin{abstract}
The ideal strength of monolayer materials possessing semimetallic, semiconducting, and insulating ground states is computed using density functional theory. Here we show that, as in graphene, a soft mode occurs at the K-point in $\mathrm{BN}$, graphane, and $\mathrm{MoS}_{2}$, while not in silicene. The transition is first-order in all cases except graphene. In BN and graphane the soft mode corresponds to a Kekulé-like distortion similar to that of graphene, while $\mathrm{MoS}_{2}$ has a distinct distortion. The phase transitions for $\mathrm{BN}$, graphane, and $\mathrm{MoS}_{2}$ are not associated with the opening of a band gap, which indicates that Fermi surface nesting is not the driving force. We perform an energy decomposition that demonstrates why the soft modes at the K-point are unique and how strain drives the phonon instability.
\end{abstract}

PACS numbers: 62.20.M-, 62.23.Kn, 63.22.-m, 64.70.Nd

Ideal strength, the maximum stress an infinite, defectfree crystal can withstand at zero temperature, is an upper limit that provides a measure for the intrinsic strength of the chemical bonding and overall stability of a material [1]. Ideal strength is ultimately dictated by what is known as the elastic instability, whereby a crystal becomes unstable with respect to a homogeneous deformation along the strain path. This scenario corresponds to an imaginary-frequency or "soft" phonon mode of vanishing wavevector $(q \rightarrow 0)$ and a maximum in the stress-strain curve. However, a finite-wavevector phonon instability, known as a soft mode, occurring at a lower stress than that of the elastic instability can also limit a material's ideal strength via the transformation to a new structure with a lower elastic instability. Acoustic phonon instabilities have been predicted to limit the ideal strength of bulk aluminum [2] and bulk silicon [3] for certain strain modes.

Monolayer materials are an optimal testbed for studying the possibility of strength-limiting soft modes since they can be fabricated with unprecedented levels of crystalline perfection. Under conditions at or close to equibiaxial strain, the mechanical failure of graphene was found to stem from an optical phonon instability at the K-point of the Brillouin zone (BZ) in which the pristine honeycomb structure distorts towards a Kekulé-like structure of isolated $\mathrm{C}_{6}$ regular hexagonal rings [4. Since this structural transformation gaps the Fermi surface by breaking the symmetry of the honeycomb structure [5, 6], it has been proposed that the soft mode in graphene is a two-dimensional (2D) manifestation of a Peierls instability [7. This has stimulated work documenting the effect of doping on the instability [8, 9]. Given that the essence of the Peierls instability arises from the properties of onedimensional systems, clearly this analogy is strained and this instability cannot solely be attributed to the Fermi surface. Nonetheless, the degree to which the gapping of the Dirac point drives the instability is an open question. Li recently found that single-layer molybdenum disulfide
$\left(\mathrm{MoS}_{2}\right)$ also exhibits a soft mode under equibiaxial strain [10], which further raises the question of the origin and generality of phonon instabilities in monolayer materials.

Here we employ density functional theory (DFT) calculations to investigate a structurally and electronically diverse set of existing 2D crystals - graphene, single-layer boron nitride (BN), graphane, $\mathrm{MoS}_{2}$, and silicene - under equibiaxial strain in order to gain insight into the nature of phonon instabilities in monolayer materials. In addition to graphene and $\mathrm{MoS}_{2}$, we find a soft mode at the $\mathrm{K}$-point for $\mathrm{BN}$ and graphane leading to mechanical failure for $\mathrm{BN}$. We show that the nature of the distortion in $\mathrm{BN}$ is completely analogous to graphene, despite the fact that BN has a large band gap. This illustrates that the Fermi surface is not the general driving force of this instability. In order to elucidate the physics of this instability, we perform a decomposition of the total energy into two terms which reasonably embody the electronic and elastic aspects of the energetics. This demonstrates the potency of the electronic term for the K-point soft mode in addition to the rapid decay of the elastic term as a function of strain.

Non-spin-polarized DFT [11, 12] calculations within the generalized gradient approximation of Perdew, Burke, and Ernzerhof [13] are performed using the Vienna $a b$ initio simulation package (VASP) 14-17. The Kohn-Sham equations are solved using a plane-wave basis set (kinetic energy cutoff of $420 \mathrm{eV}$ for $\mathrm{MoS}_{2}$ and silicene, $450 \mathrm{eV}$ for graphene and graphane, and 500 $\mathrm{eV}$ for $\mathrm{BN}$ ) and the projector augmented wave method [18, 19] with soft projectors for B, C, and N. The primitive unit cell in-plane lattice vectors are chosen to be $\mathbf{a}_{\mathbf{1}}=\sqrt{3} l / 2 \hat{\mathbf{x}}-3 l / 2 \hat{\mathbf{y}}$ and $\mathbf{a}_{\mathbf{2}}=\sqrt{3} l / 2 \hat{\mathbf{x}}+3 l / 2 \hat{\mathbf{y}}$, where $l$ is the in-plane length of the nearest-neighbor C-C, B-N, $\mathrm{Mo-S}$, and $\mathrm{Si}-\mathrm{Si}$ bond for graphene and graphane, $\mathrm{BN}$, $\mathrm{MoS}_{2}$, and silicene, respectively. The in-plane lattice vectors of the $\mathrm{K}$-cell supercell [4 commensurate with a K-point lattice distortion are $\mathbf{A}_{\mathbf{1}}=2 \mathbf{a}_{\mathbf{1}}+\mathbf{a}_{\mathbf{2}}$ and $\mathbf{A}_{\mathbf{2}}=\mathbf{a}_{\mathbf{1}}+2 \mathbf{a}_{\mathbf{2}}$. The out-of-plane lattice vector length 


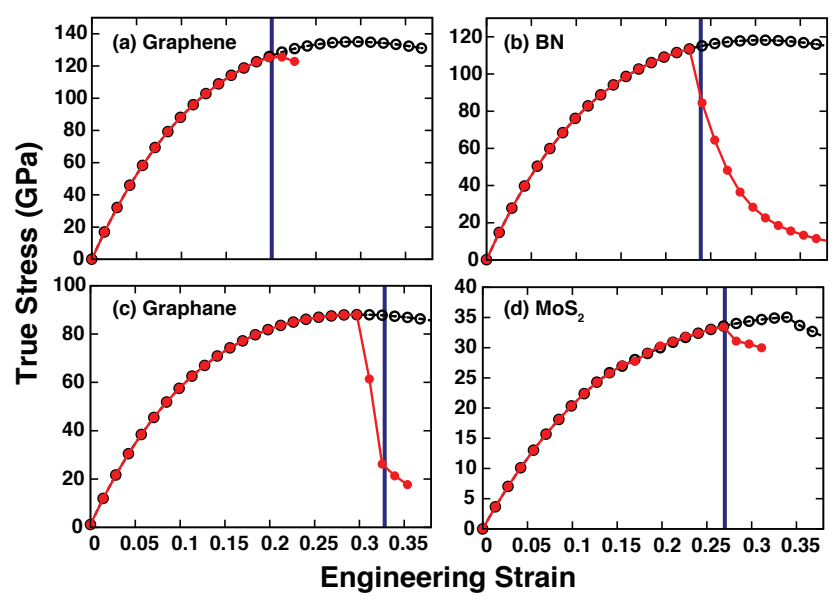

FIG. 1. True stress $\sigma$ vs. engineering strain $\varepsilon$ for (a) graphene, (b) BN, (c) graphane, and (d) $\mathrm{MoS}_{2}$ under equibiaxial strain. Black lines and open circles for the primitive unit cell; red lines and filled circles for the K-cell. The strain at which a phonon mode goes soft at the K-point is indicated by a blue line.

is chosen to be $14 \AA$ for graphene, BN, graphane, and silicene and $16 \AA$ for $\mathrm{MoS}_{2}$. To sample reciprocal space we employ $k$-point grids of $20 \times 20 \times 1$ for $\mathrm{MoS}_{2}$ and silicene and $24 \times 24 \times 1$ for graphene, graphane, and $\mathrm{BN}$ for the primitive cell and $8 \times 8 \times 1$ for graphene, $\mathrm{BN}$, and $\mathrm{MoS}_{2}$ and $9 \times 9 \times 1$ for graphane for the K-cell. The total energy, ionic positions, and stress tensor components are converged to $10^{-6} \mathrm{eV}, 0.01 \mathrm{eV} / \AA$, and $10^{-3}$ $\mathrm{GPa}$, respectively. Phonons at the K-point are obtained using the frozen phonon method. To compute stressstrain curves the unit cell is equibiaxially strained, ionic positions are perturbed to allow symmetry breaking, and then the ions are fully relaxed. We renormalize the equibiaxial true stress $\sigma=\left(\sigma_{x x}+\sigma_{y y}\right) / \sqrt{2}$ of each 2D material to the interlayer spacing of the most closely related bulk material 20] to give a physical reference for stress values. Density functional perturbation theory 21] calculations in the QUANTUM ESPRESSO package [22] are performed with a $10 \times 10 \times 1 q$-point grid for the initial search for soft modes as a function of equibiaxial strain.

For BN and graphane, in addition to graphene [4] and $\mathrm{MoS}_{2}$ [10, under equibiaxial strain the first instance of the eigenvalues of a phonon branch becoming imaginary at a finite wavevector occurs at the K-point. The critical values of equibiaxial engineering strain $\varepsilon=\left(\varepsilon_{x x}+\right.$ $\left.\varepsilon_{y y}\right) / \sqrt{2}$ at which the phonon mode goes soft at the Kpoint computed via the frozen phonon method are 0.201 , $0.239,0.328$, and 0.270 for graphene, BN, graphane, and $\mathrm{MoS}_{2}$, respectively. No finite-wavevector soft modes preceding the elastic instability are found for silicene.

To explore the impact of the K-point soft mode on the ideal strength, in Fig. 1 we compare the stress-strain curve of the K-cell commensurate with a K-point lattice distortion to that of the primitive cell. At critical values of strain identical or close to those found via the frozen phonon method, the K-cell curves shown in red significantly deviate from the primitive cell curves shown in black in the form of a drop in stress associated with a transformation to a new structure with a lower elastic instability. Computing the stress-strain curve on a finer grid of strain values near this transformation and tracking the changes in the relaxed ionic positions reveals that while the phase transition of graphene is continuous, $\mathrm{BN}$, graphane, and $\mathrm{MoS}_{2}$ each undergo a first-order phase transition with a sharp discontinuity in the stress and bond lengths. The first-order nature is most apparent in graphane, for which the distorted structure becomes (a)

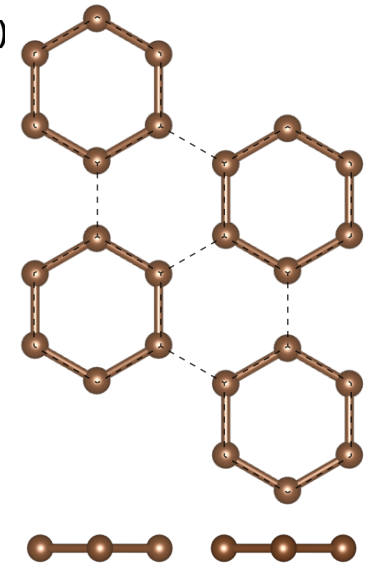

(c)

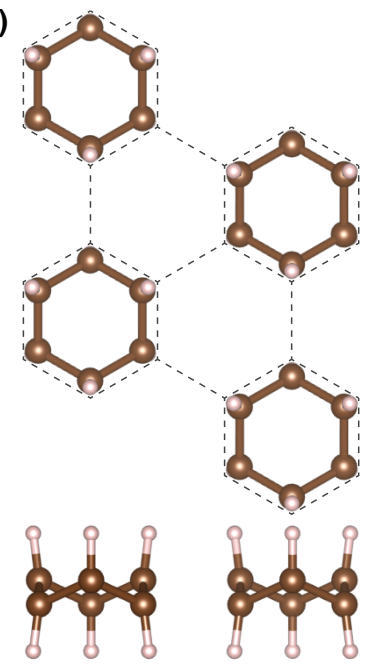

(b)

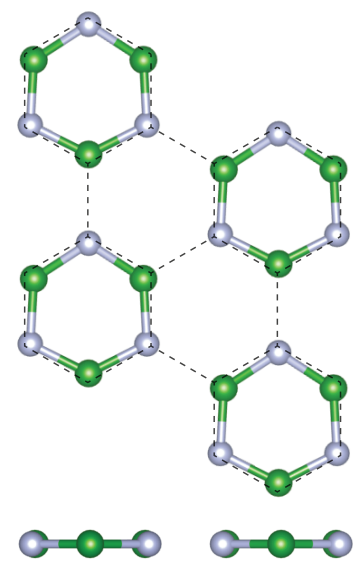

(d)

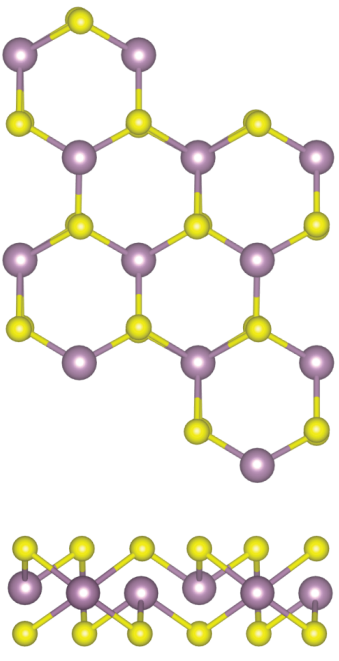

FIG. 2. Top and side orthographic projections of the distorted structures for (a) graphene, (b) BN, (c) graphane, and (d) $\mathrm{MoS}_{2}$ at equibiaxial strains of $0.212,0.240,0.328$, and 0.270 , respectively. The $\mathrm{C}, \mathrm{B}, \mathrm{N}, \mathrm{H}, \mathrm{Mo}$, and $\mathrm{S}$ atoms are represented as brown, green, silver, white, purple, and yellow spheres, respectively. Dashed lines indicate the undistorted strained lattice. 
(a) Graphene

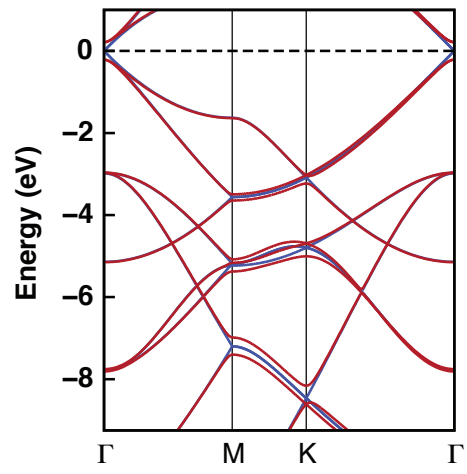

(b) BN

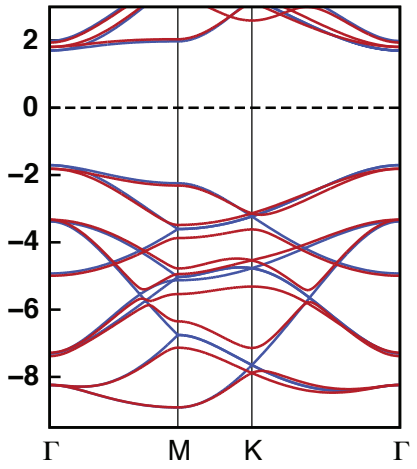

(c) Graphane

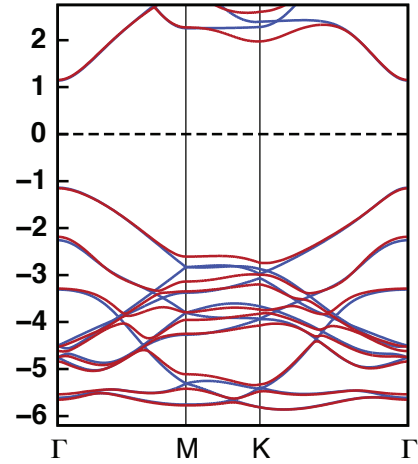

(d) $\mathrm{MoS}_{2}$

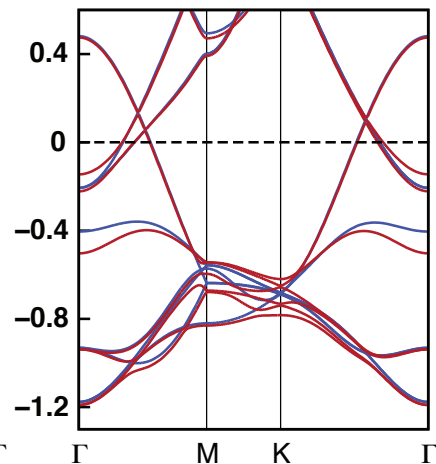

FIG. 3. K-cell electronic band structures for (a) graphene, (b) BN, (c) graphane, and (d) MoS 2 at equibiaxial strains of $0.212,0.240,0.328,0.270$, respectively. Blue lines for the undistorted structure; red lines for the partially-distorted structure corresponding to a 50\%/50\%,60\%/40\%,90\%/10\%, and 25\%/75\% linear combination of the undistorted/distorted structures for graphene, $\mathrm{BN}$, graphane, and $\mathrm{MoS}_{2}$, respectively. The $k$-point labels $\Gamma, \mathrm{M}$, and $\mathrm{K}$ correspond to the center, edge midpoint, and corner of the BZ and the dashed black line indicates the band gap midpoint for insulators and the Fermi energy for MoS ${ }_{2}$.

the ground state noticeably before the phonon goes soft. The elastic instability, corresponding to the peak of the primitive cell curves, occurs at a strain (stress) of 0.297 (135.0 GPa), $0.311(118.2 \mathrm{GPa}), 0.297(88.0 \mathrm{GPa})$, and 0.339 (35.0 GPa) for graphene, $\mathrm{BN}$, graphane, and $\mathrm{MoS}_{2}$, respectively. The ideal strength of graphene, $\mathrm{BN}$, and $\mathrm{MoS}_{2}$ are limited by the phonon instabilities since they correspond to substantially reduced strain (stress) values of $0.206(125.9 \mathrm{GPa}), 0.231$ (114.1 GPa), and 0.269 $(33.4 \mathrm{GPa})$, respectively. In contrast, for graphane the phonon instability does not precede the elastic instability so we do not predict the ideal strength is reduced by the K-point soft mode.

The distorted structures that result from the soft modes are illustrated in Fig. 2 Like in the case of graphene, the soft mode has a $2 \mathrm{D}$ irreducible representation and anharmonicity determines the minimumenergy direction and hence the ground-state structure [4]. Graphene, BN, and graphane distort towards Kekulélike structures consisting of isolated units of $\mathrm{C}_{6}$ regular hexagons for graphene, $\mathrm{B}_{3} \mathrm{~N}_{3}$ irregular hexagons for $\mathrm{BN}$, and buckled $\mathrm{C}_{6} \mathrm{H}_{6}$ structures similar to that of the chair conformation of cyclohexane (without the equatorial $\mathrm{H}$ atoms) for graphane. Such distortions have a beautiful classical analogy in strained porous elastomeric sheets, whose failure modes under equibiaxial strain correspond to arrays of alternating smaller and larger pores [23. $\mathrm{MoS}_{2}$ undergoes a distinct structural transformation in which Mo and S atoms move out-of- and in-plane, respectively. In the distorted structure one of the three Mo sites has six nearly-equal Mo-S nearest-neighbor bond lengths, and two of the three Mo sites distort (one in the $+\hat{\mathbf{z}}$ direction and one in the $-\hat{\mathbf{z}}$ direction, where $\hat{\mathbf{z}}$ is the out-ofplane direction) towards trigonal pyramidal coordination with three nearest-neighbor S atoms.
To investigate the nature and mechanism of the phonon instabilities, in Fig. 3 we examine the K-cell electronic band structures with and without distortion at critical strain, i.e., strained at or just beyond the onset of the soft phonon mode. The particular amounts of distortion, which correspond to a $50 \% / 50 \%, 60 \% / 40 \%$, $90 \% / 10 \%$, and $25 \% / 75 \%$ linear combination of the undistorted/distorted structures for graphene, BN, graphane, and $\mathrm{MoS}_{2}$, respectively, are chosen to most clearly illustrate how the soft mode affects the electronic bands. For graphene (Fig. 3a) a gap opens at the $\Gamma$-point, corresponding to the $\mathrm{K}$-point of the primitive cell due to zone folding, consistent with the Peierls instability picture. However, there are also numerous nonlinear splittings of degenerate bands at lower energy in graphene as well as in $\mathrm{BN}$, graphane, and $\mathrm{MoS}_{2}$. The structural distortions tend to break degeneracies and disentangle groups of bands. In some cases, such as in $\mathrm{MoS}_{2}$ (Fig. 3 3 ), specific bands substantially shift towards lower energy in parts of the BZ. BN (Fig. 3b) and graphane (Fig. 3p) are insulating in the undistorted state with substantial gaps of $3.4 \mathrm{eV}$ and $3.3 \mathrm{eV}$, respectively. While for $\mathrm{BN}$ the distorted structure remains insulating, for graphane after the onset of the phonon instability the fully distorted structure (whose bands are not shown) is semimetallic. For $\mathrm{MoS}_{2}$, a semiconductor in its unstrained state that becomes semimetallic at an equibiaxial strain of approximately 0.13 [10, 24], the structural distortion does not open up a gap as indicated by the multiple bands passing through the Fermi energy for the partially-distorted structure. Since the soft mode distortion is not accompanied by a band gap opening for BN, graphane, and $\mathrm{MoS}_{2}$, it is clear that a 2D analogy to the Peierls distortion cannot be the underlying mechanism in general. Furthermore, the fact that $\mathrm{BN}$ and graphane have sub- 
stantial band gaps and exhibit very similar soft modes to that of graphene strongly suggests that for graphene the gapping of the Dirac point is more of a consequence than a cause of the phonon instability.

In order to further elucidate the mechanism of the phonon instabilities and to quantitatively examine different effects, we introduce a scheme to partition the total energy in order to compare the two most electronically disparate cases: graphene (a semimetal) and $\mathrm{BN}$ (an insulator with a large gap). The total energy expression in DFT can be written as a function of the Kohn-Sham eigenvalues:

$$
\begin{aligned}
E_{t o t} & =\sum_{i, \mathbf{k}} \epsilon_{i \mathbf{k}} \theta\left(\epsilon_{F}-\epsilon_{i \mathbf{k}}\right)-\frac{1}{2} \int \frac{\rho(\mathbf{r}) \rho\left(\mathbf{r}^{\prime}\right)}{\left|\mathbf{r}-\mathbf{r}^{\prime}\right|} d^{3} r d^{3} r^{\prime} \\
& +E_{x c}[\rho]-\int v_{x c}(\mathbf{r}) \rho(\mathbf{r}) d^{3} r+E_{\text {nuc }}
\end{aligned}
$$

where $\epsilon_{i \mathbf{k}}$ is the Kohn-Sham eigenvalue of the $i$ th band at $k$-point $\mathbf{k}, \epsilon_{F}$ is the Fermi energy, $\rho(\mathbf{r})$ is the charge density at position $\mathbf{r}, E_{x c}$ and $v_{x c}$ are the exchangecorrelation energy and potential, respectively, and $E_{n u c}$ is the electrostatic energy of the nuclei. In the spirit of previous work on Fermi surface nesting [25, 26], we partition $E_{\text {tot }}$ into an "electronic" band energy $E_{\text {elec }}$ and an "elastic" energy $E_{\text {elas }}$ defined as follows:

$$
\begin{aligned}
& E_{\text {elec }}=\sum_{i, \mathbf{k}}\left(\epsilon_{i \mathbf{k}}-\epsilon_{a}\right) \theta\left(\epsilon_{F}-\epsilon_{i \mathbf{k}}\right) \\
& E_{\text {elas }}=E_{\text {tot }}-E_{\text {elec }}
\end{aligned}
$$

where a reference "anchor" $\epsilon_{a}$ from which to measure the Kohn-Sham eigenvalues is necessary since there is an arbitrary constant in the treatment of an infinite interacting system related to individually divergent summations [27. For the anchor we choose the average of the highest occupied and lowest unoccupied eigenvalues, e.g. the Dirac point states for graphene, which remain stationary to first order for a non-interacting system. The band structures in Fig. 3a-c are plotted with respect to this anchor choice.

In Fig. 4 we plot $E_{\text {elec }}$ and $E_{\text {elas }}$ as a function of the soft mode distortion amplitude for unstrained and critically-strained graphene and BN. It should be noted that only the quadratic regime is relevant in terms of deducing the instability. For graphene (Fig. 4 4 ) and BN (Fig. 4 b) $E_{\text {elec }}$ is negative, indicating that changes in band energy drive the soft mode transitions. Since BN is insulating, this demonstrates that band energy lowering can be appreciable in such phase transitions even in the absence of a Fermi surface [28]. For graphene we performed this partition for all the other modes at the K-point in addition to selected modes at the M-point (not shown), and no other mode had such a large, negative quadratic coefficient. Every negative electronic term was at least 2-3 times smaller in magnitude. For both (a) Graphene

(b) $\mathrm{BN}$

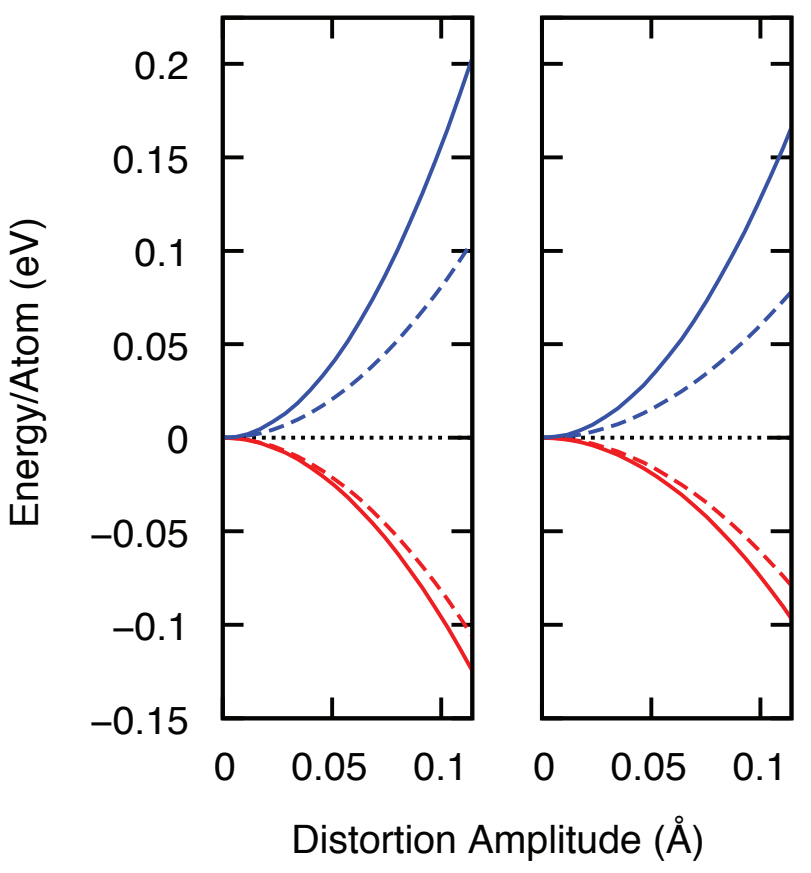

FIG. 4. Change in electronic (red) and elastic (blue) energies as a function of soft mode distortion amplitude for the K-cell structures of (a) graphene and (b) BN at equibiaxial strains (dashed lines) of 0.212 and 0.240 , respectively, compared to that at zero strain (solid lines).

graphene and BN the magnitude of the band energy lowering decreases with strain, and therefore strain actually weakens the electronic driving force despite the fact that it is essential for triggering the transition. The phonon instability emerges since $E_{\text {elas }}$ decays much more rapidly as a function of strain. Therefore, the key role of strain is to soften the elastic term such that the electronic term can dominate and drive the total energy negative. For both graphene and $\mathrm{BN}$ it is this strain-induced softening of the elastic term that enables the soft mode. Important future work will be building a physical understanding of how and why particular modes at the K-point have such a strong electronic term.

In conclusion, using DFT calculations we find soft modes similar to that of graphene for $\mathrm{BN}$, graphane, and $\mathrm{MoS}_{2}$ that limit the ideal strength of $\mathrm{BN}$ and $\mathrm{MoS}_{2}$ under equibiaxial strain. While for $\mathrm{BN}$ and graphane the soft mode corresponds to a Kekulé-like distortion similar to that of graphene, $\mathrm{MoS}_{2}$ has a distinct soft mode in which $2 / 3$ of the Mo sites distort towards trigonal pyramidal coordination. The structural transitions for BN, graphane, and $\mathrm{MoS}_{2}$ are not associated with the opening of a band gap, which reveals that Fermi surface nesting does not generally play a role in these transitions. Decomposing the total energy elucidates the complementary roles of a 
large band energy lowering that decays slowly with strain and a rapidly-decaying elastic energy penalty in driving phonon instabilities in monolayer materials.

The authors acknowledge support from the National Science Foundation (Grant No. CMMI-0927891) and the New York Center for Computational Sciences at Stony Brook University/Brookhaven National Laboratory which is supported by the U.S. Department of Energy (Grant No. DE-AC02-98CH10886) and the State of New York. E.B.I. gratefully acknowledges financial support from the U.S. Department of Energy Computational Science Graduate Fellowship (Grant No. DEFG02-97ER25308).

* ebi2104@columbia.edu

† chris.marianetti@columbia.edu

[1] A. Kelly and N. Macmillan, Strong Solids (Clarendon Press, 1986)

[2] D. M. Clatterbuck, C. R. Krenn, M. L. Cohen, and J. W. Morris, Phys. Rev. Lett. 91, 135501 (2003)

[3] S. M.-M. Dubois, G.-M. Rignanese, T. Pardoen, and J.C. Charlier, Phys. Rev. B 74, 235203 (2006)

[4] C. A. Marianetti and H. G. Yevick, Phys. Rev. Lett. 105, $245502(2010)$

[5] G. W. Semenoff, Phys. Rev. Lett. 53, 2449 (1984)

[6] C.-Y. Hou, C. Chamon, and C. Mudry, Phys. Rev. Lett. 98, 186809 (2007)

[7] S.-H. Lee, H.-J. Chung, J. Heo, H. Yang, J. Shin, U.-I. Chung, and S. Seo, ACS Nano 5, 2964 (2011)

[8] C. Si, W. Duan, Z. Liu, and F. Liu, Phys. Rev. Lett. 109, 226802 (2012)

[9] S. J. Woo and Y.-W. Son, Phys. Rev. B 87, 075419 (2013)

[10] T. Li, Phys. Rev. B 85, 235407 (2012)

[11] P. Hohenberg and W. Kohn, Phys. Rev. 136, B864 (1964)
[12] W. Kohn and L. J. Sham, Phys. Rev. 140, A1133 (1965)

[13] J. P. Perdew, K. Burke, and M. Ernzerhof, Phys. Rev. Lett. 77, 3865 (1996)

[14] G. Kresse and J. Hafner, Phys. Rev. B 49, 14251 (1994)

[15] G. Kresse and J. Hafner, Phys. Rev. B 47, 558 (1993)

[16] G. Kresse and J. Furthmüller, Phys. Rev. B 54, 11169 (1996)

[17] G. Kresse and J. Furthmüller, Comput. Mater. Sci. 6, 15 (1996)

[18] P. E. Blöchl, Phys. Rev. B 50, 17953 (1994)

[19] G. Kresse and D. Joubert, Phys. Rev. B 59, 1758 (1999)

[20] We choose $3.35 \AA$ [29], $3.33 \AA$ [30, and $6.15 \AA$ [31] for graphene and graphane, $\mathrm{BN}$, and $\mathrm{MoS}_{2}$, respectively.

[21] S. Baroni, S. de Gironcoli, A. Dal Corso, and P. Giannozzi, Rev. Mod. Phys. 73, 515 (2001)

[22] P. Giannozzi et al., J. Phys. Condens. Matter 21, 395502 (2009)

[23] J. Michel, O. Lopez-Pamies, P. Ponte Castañeda, and N. Triantafyllidis, J. Mech. Phys. Solids 55, 900 (2007)

[24] E. Scalise, M. Houssa, G. Pourtois, V. Afanasev, and A. Stesmans, Nano Res. 5, 43 (2012)

[25] J. W. Mintmire and C. T. White, Phys. Rev. B 35, 4180 (1987)

[26] J. Ashkenazi, W. E. Pickett, H. Krakauer, C. S. Wang, B. M. Klein, and S. R. Chubb, Phys. Rev. Lett. 62, 2016 (1989)

[27] R. M. Martin, Electronic Structure: Basic Theory and Practical Methods (Cambridge University Press, New York, 2008)

[28] M. D. Johannes and I. I. Mazin, Phys. Rev. B 77, 165135 (2008)

[29] Y. Baskin and L. Meyer, Phys. Rev. 100, 544 (1955)

[30] L. Liu, Y. P. Feng, and Z. X. Shen, Phys. Rev. B 68, $104102(2003)$

[31] T. Böker, R. Severin, A. Müller, C. Janowitz, R. Manzke, D. Voß, P. Krüger, A. Mazur, and J. Pollmann, Phys. Rev. B 64, 235305 (2001) 